\title{
A q-analog of Ljunggren's binomial congruence
}

\section{Armin Straub ${ }^{1 / \dagger}$}

${ }^{1}$ Tulane University, New Orleans, LA, USA

Abstract. We prove a $q$-analog of a classical binomial congruence due to Ljunggren which states that

$$
\left(\begin{array}{l}
a p \\
b p
\end{array}\right) \equiv\left(\begin{array}{l}
a \\
b
\end{array}\right)
$$

modulo $p^{3}$ for primes $p \geqslant 5$. This congruence subsumes and builds on earlier congruences by Babbage, Wolstenholme and Glaisher for which we recall existing $q$-analogs. Our congruence generalizes an earlier result of Clark.

Résumé. Nous démontrons un $q$-analogue d'une congruence binomiale classique de Ljunggren qui stipule:

$$
\left(\begin{array}{l}
a p \\
b p
\end{array}\right) \equiv\left(\begin{array}{l}
a \\
b
\end{array}\right)
$$

modulo $p^{3}$ pour $p$ premier tel que $p \geqslant 5$. Cette congruence s'inspire d'une précédente congruence prouvée par Babbage, Wolstenholme et Glaisher pour laquelle nous présentons les $q$-analogues existantes. Notre congruence généralise un précédent résultat de Clark.

Keywords: $q$-analogs, binomial coefficients, binomial congruence

\section{Introduction and notation}

Recently, $q$-analogs of classical congruences have been studied by several authors including (Cla95), (And99), (SP07), (Pan07), (CP08), (Dil08). Here, we consider the classical congruence

$$
\left(\begin{array}{l}
a p \\
b p
\end{array}\right) \equiv\left(\begin{array}{l}
a \\
b
\end{array}\right) \quad \bmod p^{3}
$$

which holds true for primes $p \geqslant 5$. This also appears as Problem 1.6 (d) in (Sta97). Congruence (1) was proved in 1952 by Ljunggren, see (Gra97), and subsequently generalized by Jacobsthal, see Remark 6 .

\footnotetext{
${ }^{\dagger}$ Partially supported by grant NSF-DMS 0713836. astraub@tulane. edu

1365-8050 @ 2011 Discrete Mathematics and Theoretical Computer Science (DMTCS), Nancy, France
} 


$$
\text { Let } \begin{aligned}
{[n]_{q}:=1+q+\ldots q^{n-1},[n]_{q} !:=} & {[n]_{q}[n-1]_{q} \cdots[1]_{q} \text { and } } \\
& \left(\begin{array}{l}
n \\
k
\end{array}\right)_{q}:=\frac{[n]_{q} !}{[k]_{q} ![n-k]_{q} !}
\end{aligned}
$$

denote the usual $q$-analogs of numbers, factorials and binomial coefficients respectively. Observe that $[n]_{1}=n$ so that in the case $q=1$ we recover the usual factorials and binomial coefficients as well. Also, recall that the $q$-binomial coefficients are polynomials in $q$ with nonnegative integer coefficients. An introduction to these $q$-analogs can be found in (Sta97).

We establish the following $q$-analog of (1):

Theorem 1 For primes $p \geqslant 5$ and nonnegative integers $a, b$,

$$
\left(\begin{array}{l}
a p \\
b p
\end{array}\right)_{q} \equiv\left(\begin{array}{l}
a \\
b
\end{array}\right)_{q^{p^{2}}}-\left(\begin{array}{c}
a \\
b+1
\end{array}\right)\left(\begin{array}{c}
b+1 \\
2
\end{array}\right) \frac{p^{2}-1}{12}\left(q^{p}-1\right)^{2} \quad \bmod [p]_{q}^{3} .
$$

The congruence (2) and similar ones to follow are to be understood over the ring of polynomials in $q$ with integer coefficients. We remark that $p^{2}-1$ is divisible by 12 for all primes $p \geqslant 5$.

Observe that (2) is indeed a $q$-analog of (1): as $q \rightarrow 1$ we recover (1).

Example 2 Choosing $p=13, a=2$, and $b=1$, we have

$$
\left(\begin{array}{l}
26 \\
13
\end{array}\right)_{q}=1+q^{169}-14\left(q^{13}-1\right)^{2}+\left(1+q+\ldots+q^{12}\right)^{3} f(q)
$$

where $f(q)=14-41 q+41 q^{2}-\ldots+q^{132}$ is an irreducible polynomial with integer coefficients. Upon setting $q=1$, we obtain $\left(\begin{array}{l}26 \\ 13\end{array}\right) \equiv 2$ modulo $13^{3}$.

Since our treatment very much parallels the classical case, we give a brief history of the congruence (1) in the next section before turning to the proof of Theorem 1 .

\section{A bit of history}

A classical result of Wilson states that $(n-1) !+1$ is divisible by $n$ if and only if $n$ is a prime number. "In attempting to discover some analogous expression which should be divisible by $n^{2}$, whenever $n$ is a prime, but not divisible if $n$ is a composite number", (Bab19), Babbage is led to the congruence

$$
\left(\begin{array}{c}
2 p-1 \\
p-1
\end{array}\right) \equiv 1 \quad \bmod p^{2}
$$

for primes $p \geqslant 3$. In 1862 Wolstenholme, (Wol62), discovered (3) to hold modulo $p^{3}$, "for several cases, in testing numerically a result of certain investigations, and after some trouble succeeded in proving it to hold universally" for $p \geqslant 5$. To this end, he proves the fractional congruences

$$
\begin{aligned}
& \sum_{i=1}^{p-1} \frac{1}{i} \equiv 0 \quad \bmod p^{2}, \\
& \sum_{i=1}^{p-1} \frac{1}{i^{2}} \equiv 0 \quad \bmod p
\end{aligned}
$$


for primes $p \geqslant 5$. Using (4) and (5) he then extends Babbage's congruence (3) to hold modulo $p^{3}$ :

$$
\left(\begin{array}{c}
2 p-1 \\
p-1
\end{array}\right) \equiv 1 \quad \bmod p^{3}
$$

for all primes $p \geqslant 5$. Note that $(6)$ can be rewritten as $\left(\begin{array}{c}2 p \\ p\end{array}\right) \equiv 2$ modulo $p^{3}$. The further generalization of (6) to (1), according to (Gra97), was found by Ljunggren in 1952. The case $b=1$ of (1) was obtained by Glaisher, (Gla00), in 1900.

In fact, Wolstenholme's congruence (6) is central to the further generalization (1). This is just as true when considering the $q$-analogs of these congruences as we will see here in Lemma 5 .

A $q$-analog of the congruence of Babbage has been found by Clark (Cla95) who proved that

$$
\left(\begin{array}{l}
a p \\
b p
\end{array}\right)_{q} \equiv\left(\begin{array}{l}
a \\
b
\end{array}\right)_{q^{p^{2}}} \bmod [p]_{q}^{2} .
$$

We generalize this congruence to obtain the $q$-analog (2) of Ljunggren's congruence (1). A result similar to (7) has also been given by Andrews in (And99).

Our proof of the $q$-analog proceeds very closely to the history just outlined. Besides the $q$-analog (7) of Babbage's congruence (3) we will employ $q$-analogs of Wolstenholme's harmonic congruences (4) and (5) which were recently supplied by Shi and Pan, (SP07):

Theorem 3 For primes $p \geqslant 5$,

$$
\sum_{i=1}^{p-1} \frac{1}{[i]_{q}} \equiv-\frac{p-1}{2}(q-1)+\frac{p^{2}-1}{24}(q-1)^{2}[p]_{q} \quad \bmod [p]_{q}^{2}
$$

as well as

$$
\sum_{i=1}^{p-1} \frac{1}{[i]_{q}^{2}} \equiv-\frac{(p-1)(p-5)}{12}(q-1)^{2} \quad \bmod [p]_{q}
$$

This generalizes an earlier result (And99) of Andrews.

\section{A $q$-analog of Ljunggren's congruence}

In the classical case, the typical proof of Ljunggren's congruence (1) starts with the Chu-Vandermonde identity which has the following well-known $q$-analog:

Theorem 4

$$
\left(\begin{array}{c}
m+n \\
k
\end{array}\right)_{q}=\sum_{j}\left(\begin{array}{c}
m \\
j
\end{array}\right)_{q}\left(\begin{array}{c}
n \\
k-j
\end{array}\right)_{q} q^{j(n-k+j)} .
$$

We are now in a position to prove the $q$-analog of (1).

Proof of Theorem 1 As in (Cla95) we start with the identity

$$
\left(\begin{array}{l}
a p \\
b p
\end{array}\right)_{q}=\sum_{c_{1}+\ldots+c_{a}=b p}\left(\begin{array}{c}
p \\
c_{1}
\end{array}\right)_{q}\left(\begin{array}{c}
p \\
c_{2}
\end{array}\right)_{q} \ldots\left(\begin{array}{c}
p \\
c_{a}
\end{array}\right)_{q} q^{p \sum_{1 \leqslant i \leqslant a}(i-1) c_{i}-\sum_{1 \leqslant i<j \leqslant a} c_{i} c_{j}}
$$


which follows inductively from the $q$-analog of the Chu-Vandermonde identity given in Theorem 4 . The summands which are not divisible by $[p]_{q}^{2}$ correspond to the $c_{i}$ taking only the values 0 and $p$. Since each such summand is determined by the indices $1 \leqslant j_{1}<j_{2}<\ldots<j_{b} \leqslant a$ for which $c_{i}=p$, the total contribution of these terms is

$$
\sum_{1 \leqslant j_{1}<\ldots<j_{b} \leqslant a} q^{p^{2} \sum_{k=1}^{b}\left(j_{k}-1\right)-p^{2}\left(\begin{array}{l}
b \\
2
\end{array}\right)}=\sum_{0 \leqslant i_{1} \leqslant \ldots \leqslant i_{b} \leqslant a-b} q^{p^{2} \sum_{k=1}^{b} i_{k}}=\left(\begin{array}{l}
a \\
b
\end{array}\right)_{q^{p^{2}}} .
$$

This completes the proof of (7) given in (Cla95).

To obtain (2) we now consider those summands in 10 which are divisible by $[p]_{q}^{2}$ but not divisible by $[p]_{q}^{3}$. These correspond to all but two of the $c_{i}$ taking values 0 or $p$. More precisely, such a summand is determined by indices $1 \leqslant j_{1}<j_{2}<\ldots<j_{b}<j_{b+1} \leqslant a$, two subindices $1 \leqslant k<\ell \leqslant b+1$, and $1 \leqslant d \leqslant p-1$ such that

$$
c_{i}=\left\{\begin{array}{l}
d \text { for } i=j_{k}, \\
p-d \text { for } i=j_{\ell}, \\
p \text { for } i \in\left\{j_{1}, \ldots, j_{b+1}\right\} \backslash\left\{j_{k}, j_{\ell}\right\}, \\
0 \text { for } i \notin\left\{j_{1}, \ldots, j_{b+1}\right\} .
\end{array}\right.
$$

For each fixed choice of the $j_{i}$ and $k, \ell$ the contribution of the corresponding summands is

$$
\sum_{d=1}^{p-1}\left(\begin{array}{l}
p \\
d
\end{array}\right)_{q}\left(\begin{array}{c}
p \\
p-d
\end{array}\right) q_{q} \sum^{p \sum_{1 \leqslant i \leqslant a}(i-1) c_{i}-\sum_{1 \leqslant i<j \leqslant a} c_{i} c_{j}}
$$

which, using that $q^{p} \equiv 1$ modulo $[p]_{q}$, reduces modulo $[p]_{q}^{3}$ to

$$
\sum_{d=1}^{p-1}\left(\begin{array}{l}
p \\
d
\end{array}\right)_{q}\left(\begin{array}{c}
p \\
p-d
\end{array}\right)_{q} q^{d^{2}}=\left(\begin{array}{c}
2 p \\
p
\end{array}\right)_{q}-[2]_{q^{p^{2}}}
$$

We conclude that

$$
\left(\begin{array}{l}
a p \\
b p
\end{array}\right)_{q} \equiv\left(\begin{array}{l}
a \\
b
\end{array}\right)_{q^{p^{2}}}+\left(\begin{array}{c}
a \\
b+1
\end{array}\right)\left(\begin{array}{c}
b+1 \\
2
\end{array}\right)\left(\left(\begin{array}{c}
2 p \\
p
\end{array}\right)_{q}-[2]_{q^{p^{2}}}\right) \bmod [p]_{q}^{3} .
$$

The general result therefore follows from the special case $a=2, b=1$ which is separately proved next.

\section{A q-analog of Wolstenholme's congruence}

We have thus shown that, as in the classical case, the congruence (2) can be reduced, via (11), to the case $a=2, b=1$. The next result therefore is a $q$-analog of Wolstenholme's congruence (6).

Lemma 5 For primes $p \geqslant 5$,

$$
\left(\begin{array}{c}
2 p \\
p
\end{array}\right)_{q} \equiv[2]_{q^{p^{2}}}-\frac{p^{2}-1}{12}\left(q^{p}-1\right)^{2} \bmod [p]_{q}^{3}
$$


Proof: Using that $[a n]_{q}=[a]_{q^{n}}[n]_{q}$ and $[n+m]_{q}=[n]_{q}+q^{n}[m]_{q}$ we compute

$$
\left(\begin{array}{c}
2 p \\
p
\end{array}\right)_{q}=\frac{[2 p]_{q}[2 p-1]_{q} \cdots[p+1]_{q}}{[p]_{q}[p-1]_{q} \cdots[1]_{q}}=\frac{[2]_{q^{p}}}{[p-1]_{q} !} \prod_{k=1}^{p-1}\left([p]_{q}+q^{p}[p-k]_{q}\right)
$$

which modulo $[p]_{q}^{3}$ reduces to (note that $[p-1]_{q}$ ! is relatively prime to $[p]_{q}^{3}$ )

$$
[2]_{q^{p}}\left(q^{(p-1) p}+q^{(p-2) p} \sum_{1 \leqslant i \leqslant p-1} \frac{[p]_{q}}{[i]_{q}}+q^{(p-3) p} \sum_{1 \leqslant i<j \leqslant p-1} \frac{[p]_{q}[p]_{q}}{[i]_{q}[j]_{q}}\right) .
$$

Combining the results (8) and (9) of Shi and Pan, (SP07), given in Theorem 3 , we deduce that for primes $p \geqslant 5$,

$$
\sum_{1 \leqslant i<j \leqslant p-1} \frac{1}{[i]_{q}[j]_{q}} \equiv \frac{(p-1)(p-2)}{6}(q-1)^{2} \quad \bmod [p]_{q} .
$$

Together with (8) this allows us to rewrite $\left[12\right.$ modulo $[p]_{q}^{3}$ as

$$
\begin{array}{r}
{[2]_{q^{p}}\left(q^{(p-1) p}+q^{(p-2) p}\left(-\frac{p-1}{2}\left(q^{p}-1\right)+\frac{p^{2}-1}{24}\left(q^{p}-1\right)^{2}\right)+\right.} \\
\left.+q^{(p-3) p} \frac{(p-1)(p-2)}{6}\left(q^{p}-1\right)^{2}\right) .
\end{array}
$$

Using the binomial expansion

$$
q^{m p}=\left(\left(q^{p}-1\right)+1\right)^{m}=\sum_{k}\left(\begin{array}{c}
m \\
k
\end{array}\right)\left(q^{p}-1\right)^{k}
$$

to reduce the terms $q^{m p}$ as well as $[2]_{q^{p}}=1+q^{p}$ modulo the appropriate power of $[p]_{q}$ we obtain

$$
\left(\begin{array}{c}
2 p \\
p
\end{array}\right)_{q} \equiv 2+p\left(q^{p}-1\right)+\frac{(p-1)(5 p-1)}{12}\left(q^{p}-1\right)^{2} \quad \bmod [p]_{q}^{3} .
$$

Since

$$
[2]_{q^{p^{2}}} \equiv 2+p\left(q^{p}-1\right)+\frac{(p-1) p}{2}\left(q^{p}-1\right)^{2} \bmod [p]_{q}^{3}
$$

the result follows.

Remark 6 Jacobsthal, see (Gra97), generalized the congruence (1) to hold modulo $p^{3+r}$ where $r$ is the $p$-adic valuation of

$$
a b(a-b)\left(\begin{array}{l}
a \\
b
\end{array}\right)=2 a\left(\begin{array}{c}
a \\
b+1
\end{array}\right)\left(\begin{array}{c}
b+1 \\
2
\end{array}\right) .
$$

It would be interesting to see if this generalization has a nice analog in the $q$-world. 


\section{Acknowledgements}

Most parts of this paper have been written during a visit of the author at Grinnell College. The author wishes to thank Marc Chamberland for his encouraging and helpful support. Partial support of grant NSF-DMS 0713836 is also thankfully acknowledged.

\section{References}

[And99] George E. Andrews. $q$-analogs of the binomial coefficient congruences of Babbage, Wolstenholme and Glaisher. Discrete Math., 204(1):15-25, 1999.

[Bab19] Charles Babbage. Demonstration of a theorem relating to prime numbers. Edinburgh Philosophical J., 1:46-49, 1819.

[Cla95] W. Edwin Clark. $q$-analogue of a binomial coefficient congruence. Internat. J. Math. and Math. Sci., 18(1):197-200, 1995.

[CP08] Robin Chapman and Hao Pan. q-analogues of Wilson's theorem. Int. J. Number Theory, 4(4):539-547, 2008.

[Dil08] Karl Dilcher. Determinant expressions for $q$-harmonic congruences and degenerate Bernoulli numbers. Electron. J. Combin., 15(1), 2008.

[Gla00] James W. L. Glaisher. Residues of binomial-theorem coefficients with respect to $p^{3}$. Quart. J. Math., Oxford Ser., 31:110-124, 1900.

[Gra97] Andrew Granville. Arithmetic properties of binomial coefficients I: Binomial coefficients modulo prime powers. CMS Conf. Proc., 20:253-275, 1997.

[Pan07] Hao Pan. A q-analogue of Lehmer's congruence. Acta Arith., 128(4):303-318, 2007.

[SP07] Ling-Ling Shi and Hao Pan. A $q$-analogue of Wolstenholme's harmonic series congruence. Amer. Math. Monthly, 114(6):529-531, 2007.

[Sta97] Richard P. Stanley. Enumerative Combinatorics, Volume 1. Cambridge University Press, 1997.

[Wol62] Joseph Wolstenholme. On certain properties of prime numbers. Quart. J. Math., Oxford Ser., 5:35-39, 1862. 